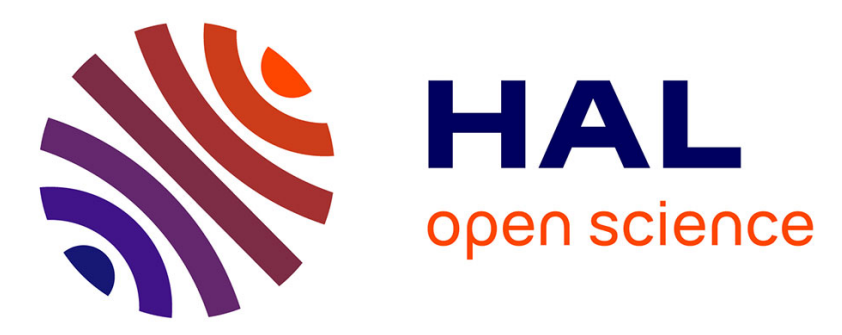

\title{
Numerical investigation of a compressible gyrofluid model for collisionless magnetic reconnection
} Luca Comisso, Daniela Grasso, Emanuele Tassi, F. Waelbroeck

\section{To cite this version:}

Luca Comisso, Daniela Grasso, Emanuele Tassi, F. Waelbroeck. Numerical investigation of a compressible gyrofluid model for collisionless magnetic reconnection. Physics of Plasmas, 2012, 19, pp.042103. 10.1063/1.3697860 . hal-00698960

\section{HAL Id: hal-00698960 https://hal.science/hal-00698960}

Submitted on 21 May 2012

HAL is a multi-disciplinary open access archive for the deposit and dissemination of scientific research documents, whether they are published or not. The documents may come from teaching and research institutions in France or abroad, or from public or private research centers.
L'archive ouverte pluridisciplinaire HAL, est destinée au dépôt et à la diffusion de documents scientifiques de niveau recherche, publiés ou non, émanant des établissements d'enseignement et de recherche français ou étrangers, des laboratoires publics ou privés. 


\title{
Numerical investigation of a compressible gyrofluid model for collisionless magnetic reconnection
}

\author{
L. Comisso, ${ }^{1}$ D. Grasso, ${ }^{1}$ E. Tassi, ${ }^{2}$ and F. L. Waelbroeck ${ }^{3}$ \\ ${ }^{1}$ Dipartimento di Energia, Politecnico di Torino, Corso Duca degli Abruzzi 24, 10129 Torino, Italy \\ and Istituto dei Sistemi Complessi-CNR, Via dei Taurini 19, 00185 Roma, Italy \\ ${ }^{2}$ Centre de Physique Théorique, CNRS-Aix-Marseille Universités, Campus de Luminy, case 907 , \\ F-13288 Marseille cedex 09, France \\ ${ }^{3}$ Institute for Fusion Studies, The University of Texas at Austin, Austin, Texas 78712-1060, USA
}

(Received 24 February 2012; accepted 9 March 2012; published online 5 April 2012)

Ion Larmor radius effects on collisionless magnetic reconnection in the presence of a guide field are investigated by means of numerical simulations based on a gyrofluid model for compressible plasmas. Compressibility along the magnetic field is seen to favour the distribution of ion guiding center density along the neutral line, rather than along the separatrices, unlike the electron density. On the other hand, increasing ion temperature reduces the intensity of localized ion guiding center flows that develop in the direction parallel to the guide field. Numerical simulations suggest that the width of these bar-shaped velocity layers scale linearly with the ion Larmor radius. The increase of ion temperature radius causes also a reduction of the electron parallel velocity. As a consequence, it is found that the cusp-like current profiles distinctive of non-dissipative reconnection are strongly attenuated. The field structures are interpreted in terms of the behavior of the four topological invariants of the system. Two of these are seen to behave similarly to invariants of simpler models that do not account for parallel ion flow. The other two exhibit different structures, partly as a consequence of the small electron/ion mass ratio. The origin of these invariants at the gyrokinetic level is also discussed. The investigation of the field structures is complemented by an analysis of the energetics of the system. (C) 2012 American Institute of Physics. [http://dx.doi.org/10.1063/1.3697860]

\section{INTRODUCTION}

Magnetic reconnection is believed to play an important role in a number of events occurring in laboratory and astrophysical plasmas, classical examples of which are sawtooth oscillations in tokamaks, magnetospheric substorms, and solar flares. ${ }^{1,2}$ Much of the progress in the understanding of magnetic reconnection has been possible thanks to the use of reduced fluid models. In such models, typically a generalized Ohm's law accounts for different mechanisms, depending on the phenomenon under consideration, that can break the frozen-in condition and allow for the reconnection of magnetic field lines. In particular, for weakly collisional plasmas, electron inertia can provide a relevant mechanism for modifying the topology of the magnetic field. A distinctive feature of reconnection mediated by electron inertia is its nondissipative nature, unlike, for instance, resistive reconnection. In particular, if other dissipative terms, possibly present in the reduced model, are omitted, one expects the resulting system to be Hamiltonian. Investigation of dissipationless magnetic reconnection has been carried out by means of Hamiltonian reduced fluid models in a number of works (see, e.g., Refs. 3 and 4 and references therein), most of which neglect ion Larmor radius effects. Investigation of Hamiltonian magnetic reconnection in the presence of finite Larmor radius (FLR) effects is then little developed, in comparison to the cold ion case. In order to fill this gap, a natural tool to consider is represented by the so-called gyrofluid models, e.g., Refs. 5-11, obtained by taking moments of the gyrokinetics equations. The Hamiltonian structure of the non-dissipative core of such models, however, is not known, except for a very few reduced versions of them.

An early investigation of reconnection by means of a Hamiltonian gyrofluid model was made in Ref. 12. In this work, the effects of ion temperature on the growth rate of the reconnecting mode and on the structures of the vorticity and of the magnetic flux function had been investigated using a reduced two-field gyrofluid model. More recently, the advanced nonlinear phase of this model has been investigated numerically, in order to elucidate the differences between the effects due to ion and sonic Larmor radius. ${ }^{13}$

A richer Hamiltonian gyrofluid model, accounting for electron inertia, was derived in Ref. 14. This model describes the evolution of ion guiding center density, electron density, and magnetic flux function. The analysis of its dispersion relation revealed also the presence of a spectral gap between the zero and the ion diamagnetic frequency. This feature represents a difference from what is observed in finite Larmor radius models valid only for $k \rho_{i} \ll 1$ (where $\rho_{i}$ is the ion Larmor radius and $k$ the modulus of the wave vector). $\mathrm{Nu}$ merical simulations of the Hamiltonian three-field gyrofluid model have been carried out in Refs. 15 and 16. These suggested that increasing $\rho_{i}$ results in a flattening of the density gradients inside the magnetic island. The presence of an equilibrium density gradients, on the other hand, has been shown to induce an island rotation at a frequency in good agreement with the asymptotic linear theory. 
A more complete Hamiltonian model has been recently derived in Ref. 17. This model, as the two and three-field models of Refs. 12 and 14, is obtained as a truncation of the gyrofluid model of Snyder and Hammett, ${ }^{9}$ but it accounts for one more moment, namely, the parallel ion flow. The Hamiltonian structure of this four-field model reveals also the presence of four Lagrangian invariants. These extend the Lagrangian invariants of the previously investigated models and naturally group into two pairs, one depending on electron fields and the other one depending on ion guiding center fields.

In this article, we present an investigation of magnetic reconnection by means of the Hamiltonian gyrofluid fourfield model. The main purpose of this investigation is to study the effect of finite ion Larmor radius on the evolution of the magnetic, density, and velocity fields, in this more complete setting, which accounts also for parallel compressibility. Numerical simulations of the evolution of such fields will be the main tool of investigation. Complementary to the direct analysis of the original variables (densities, velocities, and magnetic fields) will be the analysis in terms of the Lagrangian invariants suggested by the Hamiltonian structure. Because these invariants are simply advected quantities, their dynamics turns out to be relatively easier to follow and predict, with respect to that of the original physical fields. In particular, we will explore the differences between the dynamics of the Lagrangian invariants associated with the electron fields and that of the invariants associated with the ion fields. Indeed, although the former can be expected to have a behavior similar to that of the two invariants $G_{ \pm}$of the two and three-field model, the dynamics of the latter has never been investigated. We will also gain some insights about the physical origin of such invariants, and of their advecting velocities, by considering the derivation of the model from the gyrokinetic Vlasov equation.

Given the Hamiltonian structure of the model, we also know the expression of the conserved total energy of the system and of the different forms of energies (kinetic, magnetic, thermal and electrostatic) contributing to it. Through this, we can obtain further information about how finite Larmor radius effects can influence the distribution of the total energy into the different channels.

We remark that reconnection caused by electron inertia has been recently investigated, by means of a threedimensional four-field gyrofluid model, in Ref. 18. In this paper, the authors describe an acceleration of the reconnecting instability growth, observed at the onset of nonlinearity.

Our paper is organized as follows. In Sec. II, we review the four-field gyrofluid model equations and carry out some analytical considerations about the zero and large Larmor radius limit. These will be of support to the analysis of the numerical simulations. Section III is devoted to the analysis of the influence of $\rho_{i}$ on the field structures. In Sec. IV, we investigate the dynamics of the Lagrangian invariants of the model and present some considerations about their origin at the gyrokinetic level. Section V contains an analysis of energy transfers during the reconnection process and their dependence on $\rho_{i}$. We conclude in Sec. VI.

\section{THE COMPRESSIBLE ELECTROMAGNETIC GYROFLUID MODEL}

We consider the model of Ref. 17 adopting the following normalized quantities:

$$
\begin{aligned}
& t=\frac{v_{A}}{L} \hat{t}, \quad x=\frac{\hat{x}}{L}, \quad d_{i, e}=\frac{\hat{d}_{i, e}}{L}, \quad \rho_{i, s}=\frac{\hat{\rho}_{i, s}}{L}, \\
& n_{i, e}=\frac{L}{\hat{d}_{i}} \frac{\hat{n}_{i, e}}{n_{0}}, \quad u_{i, e}=\frac{L}{\hat{d}_{i}} \frac{\hat{u}_{i, e}}{v_{A}}, \quad A=\frac{\hat{A_{\|}}}{B L}, \quad \phi=\frac{\hat{\phi}}{B L v_{A}},
\end{aligned}
$$

where the carets denote dimensional quantities. In (1), $L$ indicates a characteristic magnetic equilibrium scale length, $v_{A}$ is the Alfvén speed based on a characteristic toroidal magnetic field intensity $B, \hat{d}_{i, e}$ are the ion and the electron skin depth, respectively, $\hat{\rho}_{s}=c_{s} / \omega_{c i}$ is the sonic Larmor radius, where $c_{s}=\left(T_{e} / m_{i}\right)^{1 / 2}$ is the sound speed and $\omega_{c i}=e B / m_{i}$ is the ion cyclotron frequency. We indicate with $\hat{\rho}_{i}=\left(T_{i} / T_{e}\right)^{1 / 2} \hat{\rho}_{s}$ the ion Larmor radius, $m_{i, e}$ are the ion and electron mass, $T_{i, e}$ the ion and electron temperature, and $n_{0}$ is a constant background density. $\hat{n}_{i, e}$ represent the ion guiding center and the electron density fluctuations, respectively, whereas $\hat{u}_{i, e}$ are the ion guiding center and electron parallel velocity. Finally, $\hat{A}_{\|}$is the parallel component of the magnetic vector potential and $\hat{\phi}$ is the electrostatic potential.

In a Cartesian coordinate system $(x, y, z)$, the equations of the Hamiltonian compressible electromagnetic gyrofluid model ${ }^{17}$ are

$$
\begin{gathered}
\frac{\partial n_{i}}{\partial t}+\left[\Phi, n_{i}\right]+\left[u_{i}, \mathcal{A}\right]=0, \\
\frac{\partial D}{\partial t}+[\Phi, D]+\tau \rho_{s}^{2}\left[n_{i}, \mathcal{A}\right]=0, \\
\frac{\partial n_{e}}{\partial t}+\left[\phi, n_{e}\right]+\left[u_{e}, A\right]=0, \\
\frac{\partial F}{\partial t}+[\phi, F]-\rho_{s}^{2}\left[n_{e}, A\right]=0,
\end{gathered}
$$

where the brackets between two generic fields $f$ and $g$ are defined by $[f, g]=\hat{\mathbf{z}} \cdot \nabla f \times \nabla g$, with $\hat{\mathbf{z}}$ the unit vector along the ignorable coordinate $z$ that is parallel to the magnetic guide field. Here, $\Phi=\Gamma_{0}^{1 / 2} \phi$ is the gyro-averaged electrostatic potential, $\mathcal{A}=\Gamma_{0}^{1 / 2} A$ is the gyro-averaged parallel magnetic potential, $D=\mathcal{A}+d_{i}^{2} u_{i}$ is the ion guiding center parallel canonical momentum, $F=A-d_{e}^{2} u_{e}$ is the electron parallel canonical momentum, whereas $\tau=T_{i} / T_{e}$ indicates the ratio between ion and electron temperatures.

We adopt the gyro-averaged operator ${ }^{6}$ in its Padé approximant form

$$
\Gamma_{0}^{1 / 2}=\left(1-\frac{\rho_{i}^{2}}{2} \nabla^{2}\right)^{-1}
$$

The system (2)-(5) is then closed by the quasi-neutrality condition

$$
n_{e}=\Gamma_{0}^{1 / 2} n_{i}+\left(\frac{\Gamma_{0}-1}{\rho_{i}^{2}}\right) \phi,
$$

where $\Gamma_{0}=\left(\Gamma_{0}^{1 / 2}\right)^{2}$, and by the Ampère's law 


$$
\nabla^{2} A=u_{e}-\Gamma_{0}^{1 / 2} u_{i}
$$

The simple form of this compressible electromagnetic gyrofluid model makes transparent the interpretation of its equations. Equation (2) is the ion continuity equation expressing the conservation of ion mass. The density of the ion guiding centers is convected by the nonlocal value of the electrostatic drift $\overline{\mathbf{v}}_{E}=\hat{\mathbf{z}} \times \nabla \Phi$. Note that the gyro-averaged electric drift is solenoidal, $\nabla \cdot \overline{\mathbf{v}}_{E}=0$, so that the compression of the ion fluid is due entirely to the last term of Eq. (2), representing the contribution of the parallel velocity of the ions to the divergence of the particle flux. Equation (3) represents the conservation of the ion parallel momentum. The first two terms form the total derivative of the ion parallel momentum taken along a path moving with velocity $\overline{\mathbf{v}}_{E}$, whereas the last term originates from the parallel ion pressure forces on a fluid element. Equations (4) and (5) are the corresponding mass and momentum conservation for the electron fluid in which gyroradius effects do not appear.

We remark that the inclusion of a non-trivial evolution for the ion guiding center density and parallel velocity breaks also the approximate "interchangeability" between $\rho_{i}$ and $\rho_{s}$ into the equations of the two-field model of Ref. 12.

The model (2)-(5) describes both the "inertial" $\left(\beta_{e} \ll m_{e} / m_{i}\right)$ and the "kinetic" $\left(\beta_{e} \gg m_{e} / m_{i}\right)$ Alfvén wave regimes ${ }^{19-21}$ in the low- $\beta_{e}$ approximation $\left(\beta_{e} \ll 1\right)$, whereas for $\beta_{e} \sim m_{e} / m_{i}$, the model equations must be modified to account for electron Landau damping. Here, the parameter that distinguishes between these regimes is $\beta_{e}=p_{e} /$ $\left(B^{2} / 2 \mu_{0}\right)=2 \rho_{s}^{2} / d_{i}^{2}$ which represents the ratio of the electron thermal pressure to the magnetic pressure. In this work, however, we consider magnetic reconnection in the kinetic Alfvén regime.

Because we are interested in the influence of the ion Larmor radius $\rho_{i}$ of the reconnection process, we consider here two extreme limits $\left(\rho_{i}=0\right.$ and $\left.\rho_{i} \rightarrow \infty\right)$ for which we can obtain some analytical estimates on the behavior of the fields.

\section{A. Cold ion limit}

In the cold ion limit $\left(\rho_{i}=0\right)$, the gyrofluid equations (2)-(5) become

$$
\begin{gathered}
\frac{\partial n_{i}}{\partial t}+\left[\phi, n_{i}\right]+\left[u_{i}, A\right]=0, \\
\frac{\partial D}{\partial t}+[\phi, D]=0, \\
\frac{\partial n_{e}}{\partial t}+\left[\phi, n_{e}\right]+\left[u_{e}, A\right]=0, \\
\frac{\partial F}{\partial t}+[\phi, F]-\rho_{s}^{2}\left[n_{e}, A\right]=0,
\end{gathered}
$$

whereas the closure relations (7) and (8) read

$$
\begin{aligned}
& n_{e}=n_{i}+\nabla^{2} \phi, \\
& \nabla^{2} A=u_{e}-u_{i} .
\end{aligned}
$$

In the absence of ion temperature, a topological conservation law becomes evident from Eq. (10) and corresponds to a "frozen-in condition" for the ion fluid canonical momentum $D$.

In the cold ion limit, there exists a rescaling that (up to terms of order $m_{e} / m_{i}$ ) puts in correspondence the gyrofluid model with the low- $\beta_{e}$ limit of the Fitzpatrick-Porcelli (FP) four-field model. ${ }^{22}$ Indeed, Eqs. (9), (11), and (13) can be linearly combined to give

$$
\frac{\partial \nabla^{2} \phi}{\partial t}+\left[\phi, \nabla^{2} \phi\right]+\left[\nabla^{2} A, A\right]=0
$$

which is the same vorticity equation appearing in the FP model. From Eqs. (10) and (12), on the other hand, we can obtain

$$
\frac{\partial u_{i}}{\partial t}+\left[\phi, u_{i}\right]+\frac{d_{e}^{2}}{d_{i}^{2}}\left(\frac{\partial u_{e}}{\partial t}+\left[\phi, u_{e}\right]\right)+\frac{\rho_{s}^{2}}{d_{i}^{2}}\left[n_{e}, A\right]=0 .
$$

Neglecting the term proportional to $d_{e}^{2} / d_{i}^{2}$ and performing the mapping $u_{i} \rightarrow u_{i} / d_{i}, n_{e} \rightarrow-Z \sqrt{2 / d_{i}^{2} \beta_{e}}$, one obtains the parallel velocity equation of the FP model in the low $-\beta_{e}$ expansion, i.e., when $\sqrt{\left(\beta_{e} / 2\right) /\left(1+\beta_{e} / 2\right)} \sim \sqrt{\beta_{e} / 2}$ (this is expected, since the gyrofluid model is derived for plasmas with $\beta_{e} \ll 1$, whereas the FP model is valid also for larger $\beta_{e}$ ).

The same rescaling for $n_{e}$ and $u_{i}$, applied to Eqs. (11) and (12), with the help of Eq. (14) and again neglecting terms of order $m_{e} / m_{i}$, yields the low- $\beta_{e}$ FP equations for the parallel magnetic perturbation $Z$ and for the poloidal flux function $A$. We observe that the terms of order $m_{e} / m_{i}$ retained in the four-field gyrofluid model are neglected in most models. We remark that discarding them, as in the FP model, seems, however, not to affect important properties, such as the existence of a Hamiltonian structure for the model.

\section{B. Large ion Larmor radius limit}

For the opposite limit of very large ion Larmor radius $\left(\rho_{i} \rightarrow+\infty\right)$, we can deduce some information, about the asymptotic behavior, from the linear quasi-neutrality condition (7) and the parallel current density expression (8).

If we assume that the fields do not diverge as $\rho_{i} \rightarrow+\infty$ (an assumption that we verify a posteriori from the simulations), then we can make use of the Ansatz

$$
\begin{aligned}
& n_{e}=n_{e 0}+\varepsilon n_{e 1}+\cdots, \\
& n_{i}=n_{i 0}+\varepsilon n_{i 1}+\cdots, \\
& \phi=\phi_{0}+\varepsilon \phi_{1}+\cdots,
\end{aligned}
$$

where $\varepsilon=1 / \rho_{i}^{2}$. At the leading order for $\varepsilon \rightarrow 0$, from Eq. (7), using the Padé approximation, we have

$$
\nabla^{4} n_{e 0}=0
$$

For our simulations, we assume periodic fields vanishing at the boundaries. Applying these conditions to Eq. (18) implies $n_{e 0}=0$. At the next order, we then have

$$
\frac{\nabla^{4}}{2} n_{e 1}=-\nabla^{2} n_{i 0}-\frac{\nabla^{4}}{2} \phi_{0}
$$


In particular, if the contribution of $n_{i}$ in the quasi-neutrality relation is negligible, this implies, at the leading order, the relation

$$
\phi \sim-\rho_{i}^{2} n_{e}
$$

By a similar argument, from the Padé approximant expression of the gyro-average operator, one obtains

$$
\Phi \sim-\frac{2}{\rho_{i}^{2}} \nabla^{-2} \phi
$$

Because of the presence of the inverse Laplacian operator, Eq. (21) shows that the gyro-averaged potential $\Phi$ is characterized by larger scales, compared to those of $\phi$. Concerning the parallel current density, one has, analogously, the following relation at the leading order:

$$
\nabla^{2} A \sim u_{e}
$$

stating that the parallel current density is brought mainly by the parallel electron velocity.

\section{ANALYSIS OF THE FIELD STRUCTURES}

We solve the system of equations (2)-(5) using a pseudospectral method on a domain $\{(x, y):-\pi \leq x<\pi$, $-2 \pi \leq y<2 \pi\}$, with a grid of $1024 \times 128$ points and imposing double periodic boundary conditions. We consider an initial equilibrium specified by

$$
n_{i_{e q}}(x)=n_{e q}, \quad n_{e_{e q}}(x)=n_{e q}, \quad A_{e q}(x)=\sum_{n=-11}^{11} a_{n} e^{i n x}
$$

where $n_{e q}$ represent a uniform, nondrifting background density, and $a_{n}$ are the Fourier coefficients of the function $f(x)$ $=0.1 / \cosh ^{2} x$. An initial perturbation

$$
\tilde{n}_{i}=\tilde{n}_{i_{0}}(\cos (x+y / 2)-\cos (x-y / 2))
$$

is added to the ion guiding center density field. The field $\phi$ is perturbed accordingly (see Eq. (7)), in such a way that the initial perturbation on $n_{e}$ is zero. We present simulations with $d_{e}=0.2, d_{i}=2$ and $\rho_{s}=0.4$, whereas $\rho_{i}$ is varied in order to investigate ion gyration effects. The corresponding equilibrium is tearing unstable with linear stability parame$\operatorname{ter}^{23} \Delta^{\prime}=59.9$. In the regime of parameters that we study, corresponding to $\Delta^{\prime} d_{e} \gg \min \left[1,\left(d_{e} / \rho_{\tau}\right)^{1 / 3}\right]$, with $\rho_{\tau}=\left(\rho_{i}^{2}+\rho_{s}^{2}\right)^{1 / 2}$, we find that our results are in agreement with the asymptotic formula $\gamma_{L} \approx 0.2 k_{y}\left(2 d_{e} \rho_{\tau}^{2} / \pi\right)^{1 / 3}$ for the linear growth rate, ${ }^{24}$ where $k_{y}=m 2 \pi / L_{y}$ is the wave vector, with $m$ integer wave number and $L_{y}$ length of the domain along the $y$ direction.

In order to facilitate the comparison between simulations obtained varying $\rho_{i}$, we show in Fig. 1 the reconnected flux $\ln \left|\delta A_{X}\right|$ as a function of the normalized time $\gamma_{L} t$, for different values of $\rho_{i}$, where the initial transient was not considered. In doing so, the nonlinear phase begins at the same normalized time, and we can compare the structures of the

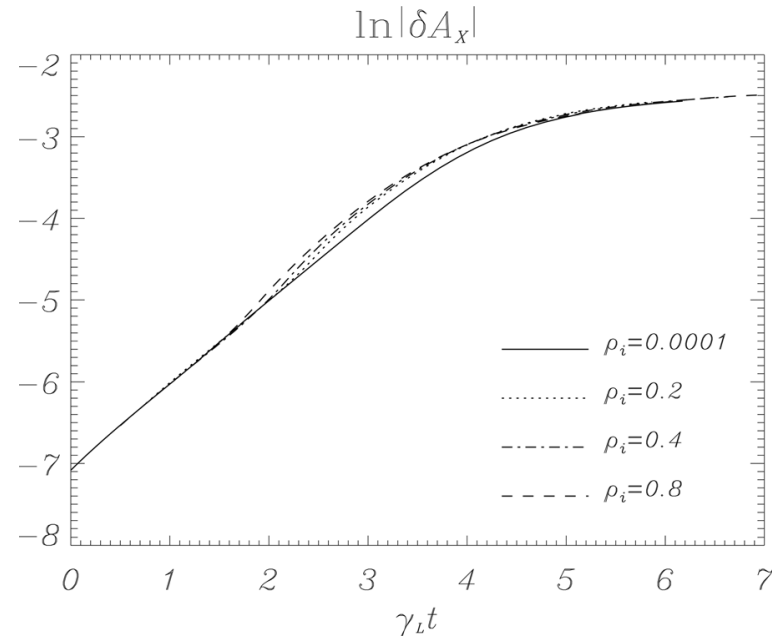

FIG. 1. Growth rate of the reconnected flux: $\ln |\delta A|$ evaluated at the X-point as a function of the normalized time $\gamma_{L} t$, for different values of $\rho_{i}$.

fields at the same level of reconnected flux, which indicates that the corresponding magnetic islands have reached the same growth level. From this figure, we also see that the super-exponential phase, a well-known characteristic of the collisionless regime, ${ }^{12,27-34}$ becomes more and more evident increasing $\rho_{i}$.

\section{A. Island shape}

As a first remark about the role of FLR effects on the field structures, we discuss their influence on the magnetic island shape. Ion gyration indeed modifies the distribution of the parallel current density $j=-\nabla^{2} A$ inside the island separatrix, which in turn is linked with the shape of the flux surfaces. We note that this is a non-constant- $A$ effect. Indeed, when $A$ is treated as a constant in $x$ over the region between the separatrices, the island shape is immutable.

Here, we compare two magnetic islands that differ in the ion Larmor radius, one with cold ions and the other with large $\rho_{i}$. Figs. 2(a) and 2(b) show their shape at the beginning of the super-exponential growth, when the reconnected flux is the same for both cases $\left(\ln \left|\delta A_{X}\right| \approx-4.2\right)$. At this stage of the island evolution, the differences in the magnetic island shape start to become particularly evident, since in the superexponential phase the island size goes from microscopic to macroscopic. The angle between the $y$-axis and the island separatrix through the X-point is greater in the hot ion case. On the other hand, the island width is greater in the cold ion limit. However, as a result of the different shape of the separatrix, the area of the island is about the same for cold and hot ions. This difference in the shape of the islands reflects the different distribution of the spectral power of the perturbed magnetic flux $\left|\delta A_{m}\right|$ at $x=0$. While the modes 0 and 1 are higher for the cold ion limit, other significant modes shown in Figs. 2(e) and 2(f) are much more relevant in the hot ion case.

In Ref. 35 it was shown, in the framework of low- $\beta$ reduced resistive MHD, that for thin $m=1$ magnetic islands, the width of the separatrix is given by the Abel transform of the inverse parallel current density. Following this approach, although in a numerical, rather than analytical framework, 

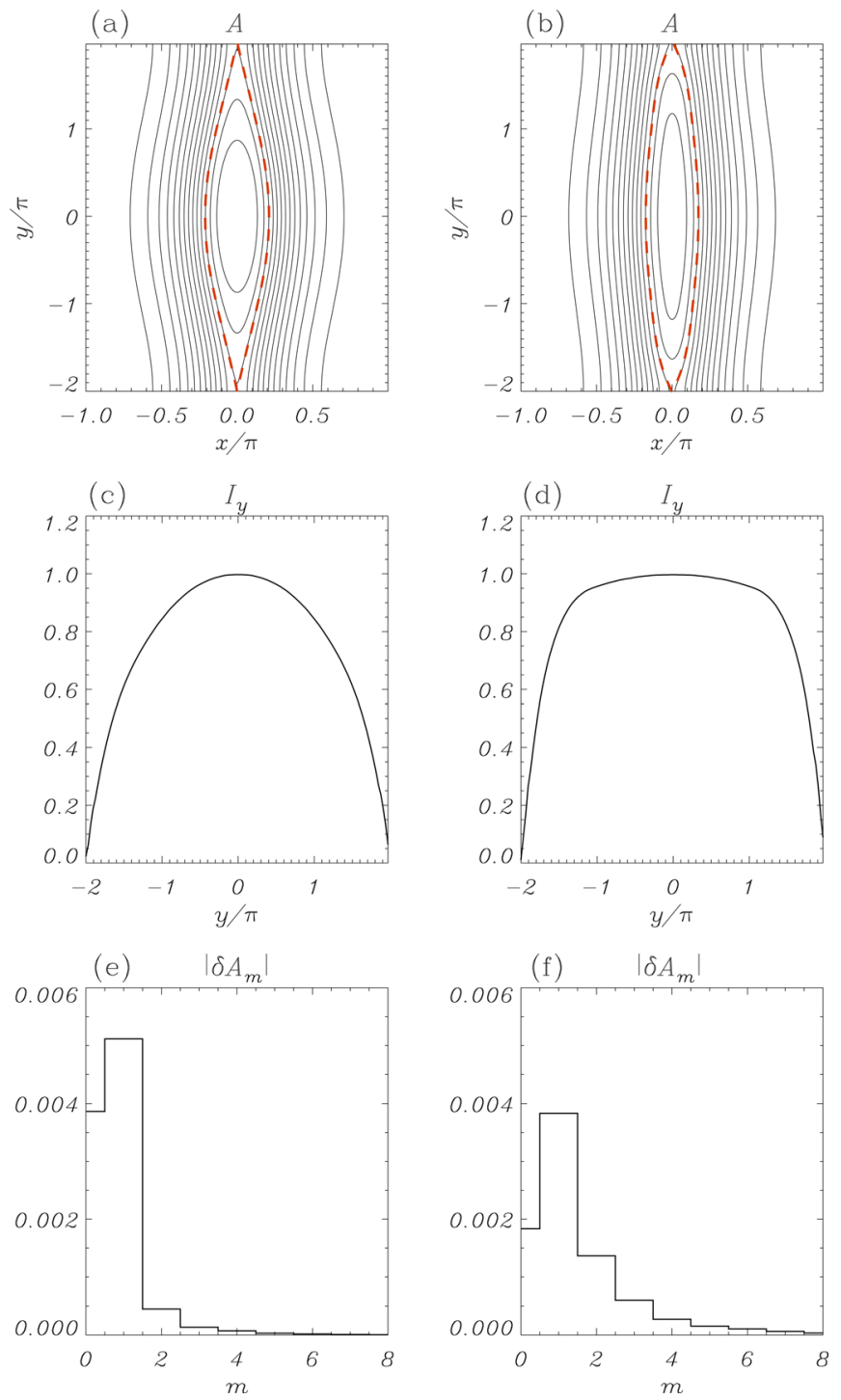

FIG. 2. Plots of the left (right) column refer to $\rho_{i}=10^{-4}\left(\rho_{i}=0.8\right)$. First row: contour plots of $A$, where the magnetic island separatrix at the corresponding time have been superimposed (red dashed line). Second row: profiles of $I_{y_{s}} / \max \left(I_{y_{s}}\right)$, where $I_{y_{s}}=\int_{-x_{s}}^{x_{s}} d x j$. Third row: perturbed magnetic flux spectrum for the first 8 modes at $x=0$. The times at which the plots refer to are $\gamma_{L} t=2.8$ for $\rho_{i}=10^{-4}$ and $\gamma_{L} t=2.5$ for $\rho_{i}=0.8$. At these normalized times $\ln \left|\delta A_{X}\right| \approx-4.2$ for both cases.

we calculate the integral of the parallel current density in the $x$ direction between the magnetic island separatrix

$$
I_{y_{s}}=-\int_{-x_{s}}^{x_{s}} d x \nabla^{2} A
$$

where $x_{s}$ is the $x$ coordinate of the island separatrix. The profile of the normalized quantity $I_{y_{s}} / \max \left(I_{y_{s}}\right)$ as a function of $y$ shown in Figs. 2(c) and 2(d) exhibits a flattening along most of the neutral line for $\rho_{i}=0.8$. This reflects the fact that the thickness of the island varies little along most of the $y$-axis.

\section{B. Parallel velocities and current density}

Figs. 3(a) and 3(b) show contour plots of $u_{i}$, for $\rho_{i}=10^{-4}$ and $\rho_{i}=0.8$ at the normalized time $\gamma_{L} t=5.5$. In the first case, the maximum gradients of $u_{i}$ are narrowed aligned along the neutral line, whereas they become broader increasing $\rho_{i}$. Moreover, for the cold ion case, these barshaped velocity layers extend deeper into the magnetic island. Their thickness $\delta_{u_{i}}$ can be evaluated from the profile of $u_{i} / \max \left(u_{i}\right)$ as a function of $x$ through the island X-point, shown in Fig. 3(c). The profiles suggest the following scaling relation:

$$
\delta_{u_{i}} \sim \rho_{i}
$$

that can be useful when evaluating the ion velocity shear across the neutral line.

Unlike what happens for ions, no bar-shaped layers along neutral line are present in the electron velocity field, regardless the value of $\rho_{i}$. Moreover, the electron velocity is largest at the magnetic island separatrix for both cold and hot ion cases. However, in the hot ion regime, narrow structures are present also in the island core, as shown in the contour plots of Fig. 4, and even better in the profiles of $u_{e}$ as a function of $x$, evaluated through the island O-point (Figs. 4(c) and 4(d)). Note that the gradients near the separatrix at $y=0$ are slightly different for $\rho_{i}=10^{-4}$ and $\rho_{i}=0.8$. On the contrary, the velocity gradients are strongly attenuated approaching the X-point for the hot ion case, as it can be appreciated by looking at the profiles of $u_{e}$ as a function of $x$, evaluated through the island X-point (Figs. 4(e) and 4(f)).

The structures in the parallel velocity fields are helpful in understanding the behavior of the parallel current density at the X-point, which we denote with $j_{X}$. We observe that the evolution of $j_{X}$ is characterized by much lower values in the hot ion regime with respect to the cold ion case. Fig. 5(top), where the perturbed current density at the X-point, $\delta j_{X}$, is plotted as a function of the normalized time $\gamma_{L} t$, illustrates that its maximum value decreases significantly when $\rho_{i}$ increases. The sharp gradients (cusp-like profiles) distinctive of collisionless reconnection ${ }^{29,31,36-39}$ remain confined in the narrow electron layer $\delta \sim d_{e}$, as shown in Fig. 5(bottom). For the various $\rho_{i}$-regimes, we observe that the influence of $u_{i}$ on $j_{X}$ is modest. In the cold ion limit, this had been already observed in Ref. 40. In order to obtain a substantial contribution of $u_{i}$ in the reconnection process, it would be required to consider large values of $\beta_{e}$ not belonging to the regime of validity of this model and also not relevant, for instance, for tokamak applications.

\section{Ion guiding center and electron densities}

The presence of compressible ion flow results in a nontrivial evolution of the ion guiding center density in comparison to what is observed in the three-field model. ${ }^{15}$ Indeed, from Fig. 6(a), one observes that $n_{i}$ develops two layers antisymmetric with respect to $x=0$ and with strong gradients along the $x$ direction. These layers form as a consequence of the four-cell pattern characterizing the stream function $\Phi$ at $\rho_{i}=0$. When ion temperature is increased, such gradients tends to flatten (Fig. 6(b)), similarly to what we have seen about the parallel velocity of the ion guiding centers. Again, 

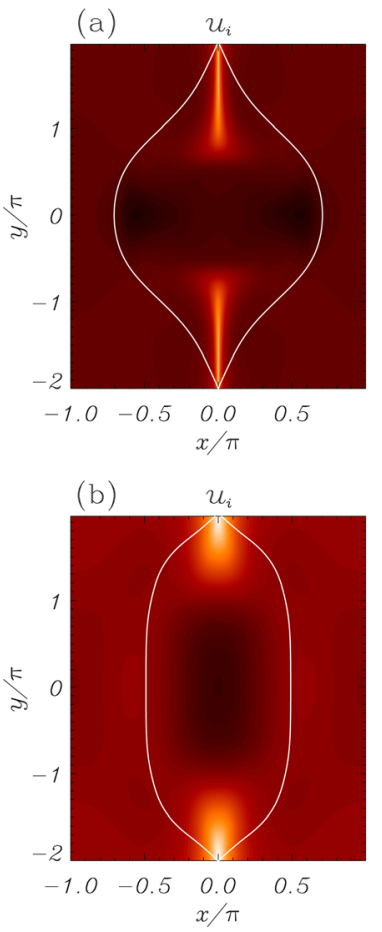

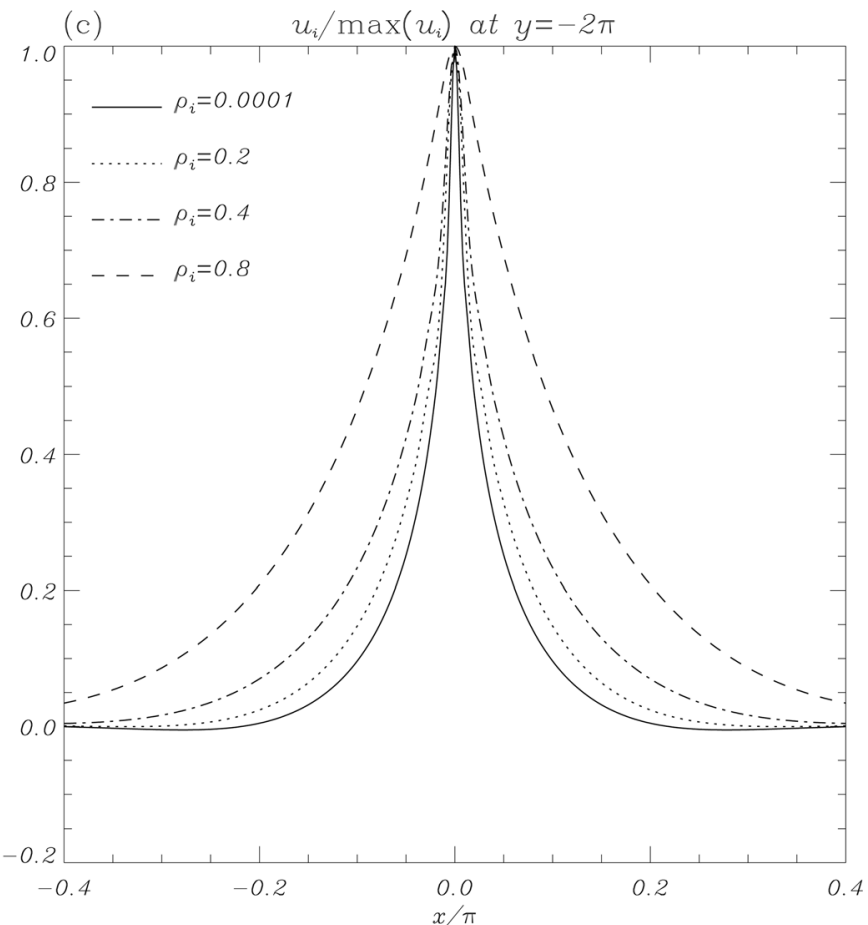

FIG. 3. Contour plots of $u_{i}$ for $\rho_{i}=10^{-4}$ (a) and for $\rho_{i}=0.8$ (b). The magnetic island separatrix at the corresponding time have been superimposed (white line). Profiles of $u_{i} / \max \left(u_{i}\right)$ as a function of $x$, evaluated at $y=-2 \pi$ (through the island X-point), for different values of $\rho_{i}$ (c). At the time which the plots refer to $\left(\gamma_{L} t=5.5\right)$, the corresponding magnetic islands have reached the same growth level. the profiles of $n_{i}$ as a function of $x$, shown in Fig. 6(c), for different values of $\rho_{i}$, help to evaluate this behavior.

We remark that because of the analogy described in Sec. II A, for $\rho_{i}=10^{-4}$ the field $n_{i}$ plays a role analogous to that of the generalized vorticity

$$
\omega=\nabla^{2} \phi+\sqrt{\frac{1+\beta_{e} / 2}{\beta_{e} / 2}} \frac{d_{i}}{d_{i}^{2}+d_{e}^{2}} Z
$$

of the FP model. Such generalized vorticity was indeed observed to develop sheets aligned with the neutral line and with strong transverse gradients, which could lead to a Kelvin-Helmholtz instability for sufficiently high $\beta_{e^{.}}{ }^{41-44}$ Note also the analogy between the definition of $\omega$ in Eq. (27) and the quasi-neutrality condition (13), from which we can make the identification $n_{i} \rightarrow-\omega$.

The evolution of $n_{e}$ and its dependence on $\rho_{i}$ (shown in Figs. 6(d)-6(f)) do not differ much from that of the 3 -field

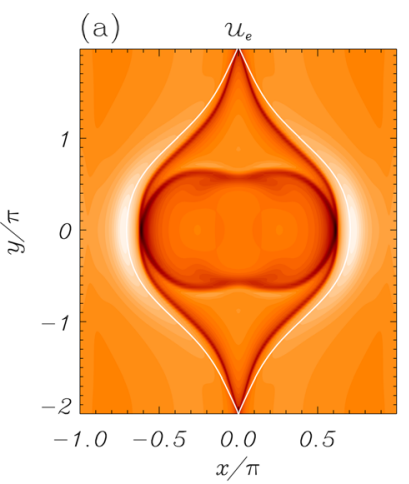

(b)

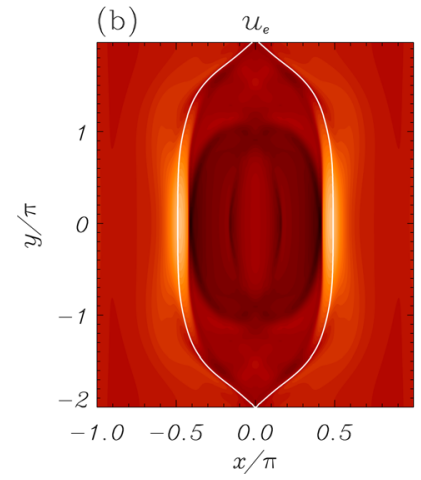

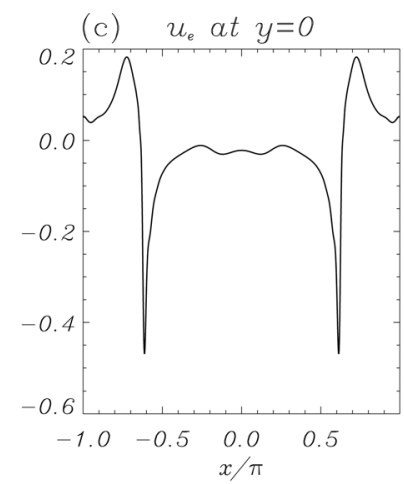

(d) $u_{e}$ at $y=0$

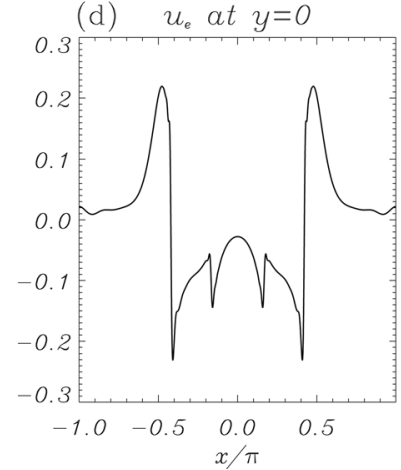

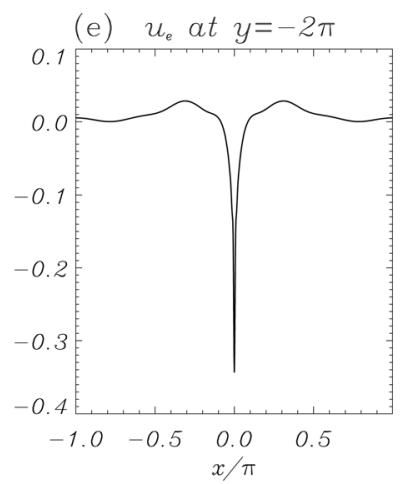

(f) $u_{e}$ at $y=-2 \pi$

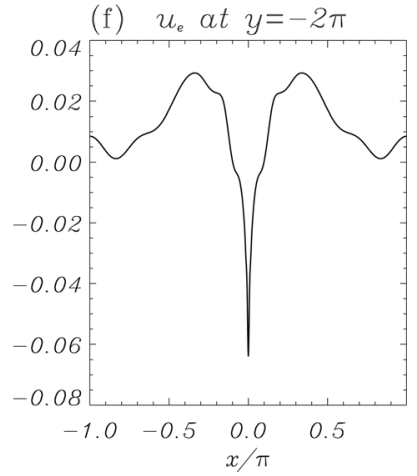

FIG. 4. Contour plots and profiles of $u_{e}$ as a function of $x$, evaluated at $y=0$ and $y=-2 \pi$, at $\gamma_{L} t=5.5$. The ion Larmor radius is $\rho_{i}=10^{-4}$ (top row) and $\rho_{i}=0.8$ (bottom row). Contour plot of the magnetic island separatrix at the corresponding time have been superimposed (white line). 

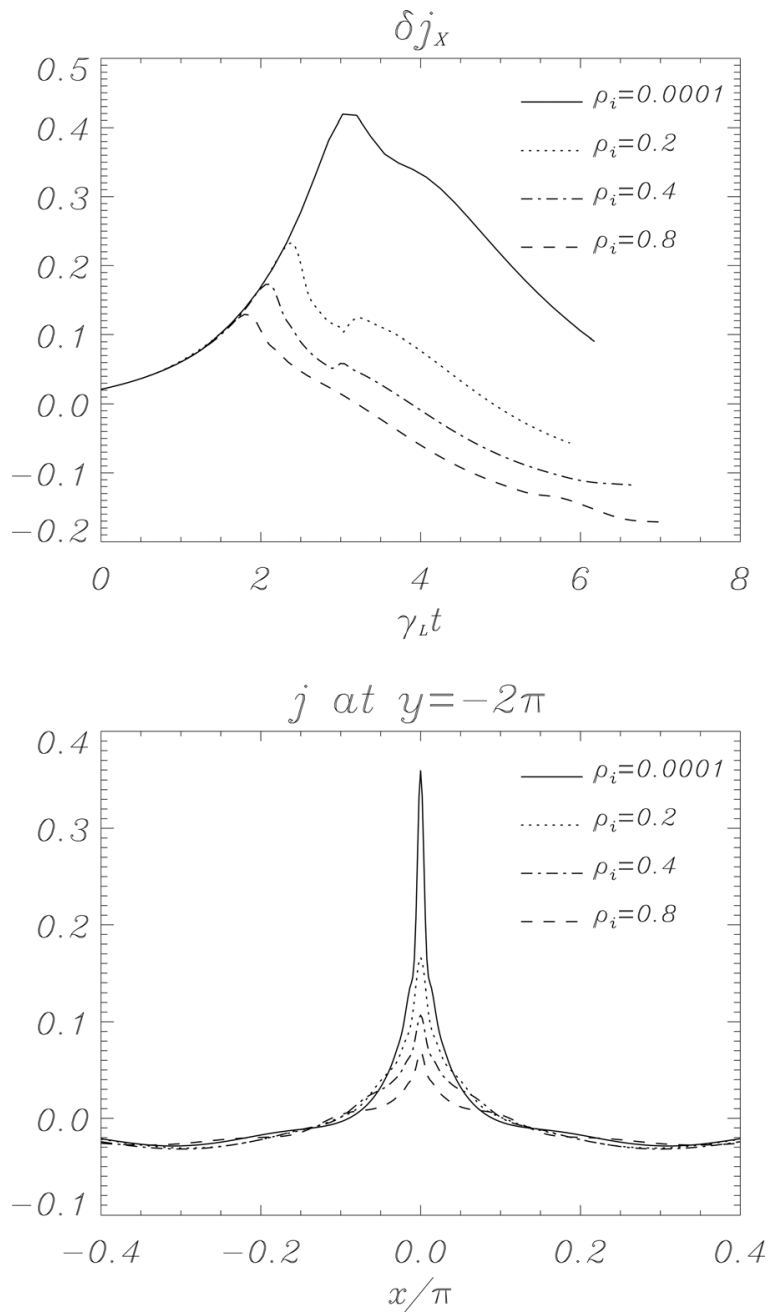

FIG. 5. Time evolution of $\delta j=j-j_{e q}$ evaluated at the X-point, for different values of $\rho_{i}$ (top). Profiles of $j=-\nabla^{2} A$ as a function of $x$, evaluated at $y=-2 \pi$ (through the island X-point), for different values of $\rho_{i}$ (bottom), at $\gamma_{L} t=5.5$.

gyrofluid model investigated in Ref. 15. This can be interpreted in terms of the behavior of two Lagrangian invariants which we will review in Sec. IV.

We remark that although $n_{i}$ has a non-trivial evolution, due to the effect of parallel compressibility, its contribution in the quasi-neutrality relation is less relevant than that of $n_{e}$, except for a thin region around the neutral line (see Fig. 6).

\section{MODEL INVARIANTS}

The system of gyrofluid equations (2)-(5) can be cast into noncanonical Hamiltonian form,${ }^{17}$ and its equations can be written in terms of four topological invariants, defined as

$$
I_{ \pm}=D \pm d_{i} \rho_{i} n_{i}, \quad G_{ \pm}=F \pm d_{e} \rho_{s} n_{e} .
$$

Indeed, using $I_{ \pm}$and $G_{ \pm}$as field variables, the gyrofluid system can be written in the following form of advection equations:

$$
\begin{gathered}
\frac{\partial I_{ \pm}}{\partial t}+\left[\Phi_{ \pm}, I_{ \pm}\right]=0, \\
\frac{\partial G_{ \pm}}{\partial t}+\left[\phi_{ \pm}, G_{ \pm}\right]=0,
\end{gathered}
$$

where

$$
\Phi_{ \pm}=\Phi \mp \frac{\rho_{i}}{d_{i}} \mathcal{A}, \quad \phi_{ \pm}=\phi \pm \frac{\rho_{s}}{d_{e}} A
$$

are stream functions of the velocity fields $\overline{\mathbf{v}}_{ \pm}=\hat{\mathbf{z}} \times \nabla \Phi_{ \pm}$ and $\mathbf{v}_{ \pm}=\hat{\mathbf{z}} \times \nabla \phi_{ \pm}$that convect the fields $I_{ \pm}$and $G_{ \pm}$, respectively.

The invariants $G_{ \pm}$and their stream functions $\phi_{ \pm}$were already present in simpler Hamiltonian models for reconnection, and their dynamics has been extensively studied in Refs. $15,25,26,33$, and 43 . The role of $I_{ \pm}$and $\Phi_{ \pm}$, on the other hand, is presented here for the first time.

\section{A. Origin of the topological invariants of the system}

The introduction of the parallel ion flow, with respect to the three-field gyrofluid model of Ref. 14, "symmetrizes" the treatment of the electron and ion dynamics. This symmetry appears evident already in the model equations (2)-(5) and occurs also in the symmetric form of the Lagrangian invariants (28) and of the corresponding stream functions (31). For the electron dynamics, the origin of such stream functions, at the kinetic level, has been investigated in Ref. 45, where the three-field model of Ref. 46 has been extended to account for a kinetic electron response, and in Refs. 47-49, where the analogy between the temporal evolution of the invariants $G_{+}$and $G_{-}$and that of the drift-kinetic distribution function of the electrons at fixed canonical momentum has been pointed out. More recently, the relation of such invariants in the context of a different hybrid fluid-kinetic model has also been discussed. ${ }^{50}$ Due to the aforementioned symmetry, there exist analogous reminiscences of the Lagrangian invariants $I_{ \pm}$and of the stream functions $\Phi_{ \pm}$at the gyrokinetic level. By following a procedure analogous to that of Refs. 45, 47-50, we can obtain information about the origin of $I_{ \pm}$and $\Phi_{ \pm}$from ion gyrokinetic equations.

We consider the ion gyrokinetic equation that Snyder and Hammett ${ }^{9}$ used as starting point for the derivation of their gyrofluid model and specify it to the hypotheses of our fourfield model (which is indeed a truncation of the model of Ref. 9). This means that we assume a gyrokinetic distribution function independent of $z$, a uniform equilibrium magnetic field directed along $\hat{\mathbf{z}}$, and no collisions. With these hypotheses, the ion gyrokinetic equation (derived from Eq. (11) of Ref. 9), in our normalization, reads

$$
\frac{\partial \mathcal{F}}{\partial t}+\left[\langle\phi\rangle-v_{\|}\langle A\rangle, \mathcal{F}\right]-\frac{1}{d_{i}}\left(\frac{\partial\langle A\rangle}{\partial t}+[\langle\phi\rangle,\langle A\rangle]\right) \frac{\partial \mathcal{F}}{\partial v_{\|}}=0,
$$

where $\mathcal{F}\left(x, y, v_{\|}, t ; \mu\right)$ is the gyrokinetic distribution function, $v_{\|}$the parallel velocity coordinate, and $\mu$ the magnetic moment which is constant in the lowest order dynamics and, therefore, appears as a parameter. The brackets \langle\rangle indicate the action of the gyroaverage operator, which, in Fourier space, corresponds to $J_{0}\left(\sqrt{2 \mu} k / \omega_{c i}\right)$.

Equation (32) appears formally the same as the electron kinetic equation of Ref. 45, provided one replaces the 

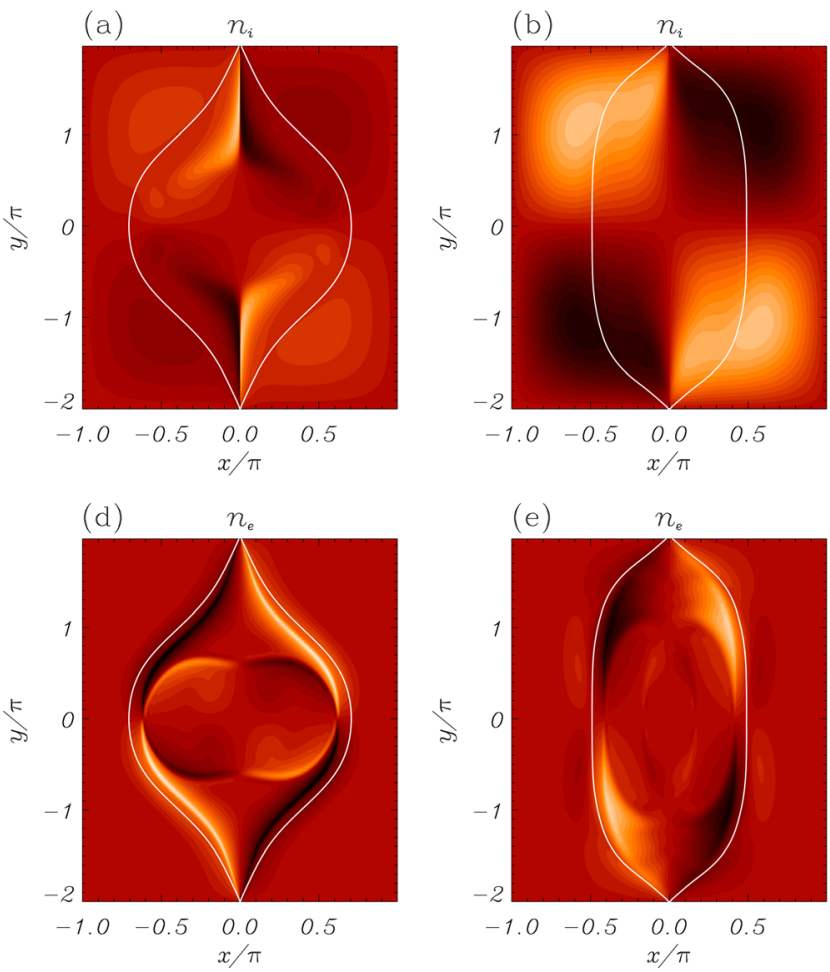

electrostatic and magnetic potential with their gyroaveraged counterparts. Consequently, Eq. (32) can be cast in the Lagrangian conservative form

$$
\frac{\partial \overline{\mathcal{F}}}{\partial t}+[\bar{\varphi}, \overline{\mathcal{F}}]=0
$$

where $\overline{\mathcal{F}}(x, y, p, t ; \mu)=\mathcal{F}\left(x, y, v_{\|}, t ; \mu\right)$ is the distribution function in terms of the canonical momentum divided by the ion mass, whose expression reads $p=v_{\|}-\langle A\rangle / d_{i}$. On the other hand, $\bar{\varphi}=\langle\phi\rangle-p\langle A\rangle-\langle A\rangle^{2} /\left(2 d_{i}\right)$ is a generalized stream function. As pointed out in Refs. 47-49, this form of the kinetic equations puts in evidence the existence, in the (gyro)kinetic description, of infinite Lagrangian invariants, labelled by constant values of $p$, each of which is advected by its own stream function. Such invariants take the place of the invariants $I_{ \pm}$of the gyrofluid model.

Consistently with the derivation by Snyder and Hammett, we write the distribution function as $\mathcal{F}=F_{0}+f$, where

$$
F_{0}\left(v_{\|} ; \mu\right)=\frac{\bar{n}_{0}}{(2 \pi)^{3 / 2} v_{t i}^{2}} \mathrm{e}^{-v_{\|}^{2} / 2 v_{t i}^{2}-\mu / v_{t i}^{2}}
$$

is a Maxwellian equilibrium distribution function, with $v_{t i}=\rho_{i} / d_{i}$ and $\bar{n}_{0}$ indicating the normalized ion thermal speed and background density, respectively. $f\left(x, y, v_{\|}, t ; \mu\right)$, on the other hand, is the perturbation. We remark that, due to our hypotheses, the time-independent part of the perturbation, which is required in the general gyrofluid model, is absent here. Applying the ordering of the gyrofluid model to Eq. (32), one finds the following governing equation for $f$ :

$$
\frac{\partial}{\partial t}\left(f+\frac{1}{d_{i}} \frac{v_{\|}}{v_{t i}^{2}} F_{0}\langle A\rangle\right)+\left[\langle\phi\rangle-v_{\|}\langle A\rangle, f+\frac{1}{d_{i}} \frac{v_{\|}}{v_{t i}^{2}} F_{0}\langle A\rangle\right]=0 .
$$

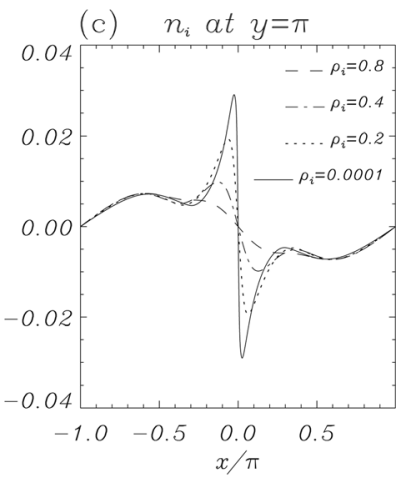

FIG. 6. Contour plots of $n_{i}$ for $\rho_{i}=10^{-4}$ (a) and $\rho_{i}=0.8$ (b), and profiles of $n_{i}$ as a function of $x$, evaluated at $y=\pi$, for different values of $\rho_{i}$ (c). Contour plots of $n_{e}$ for $\rho_{i}=10^{-4}$ (d) and $\rho_{i}=0.8(\mathrm{e})$, and profiles of $n_{e}$ as a function of $y$, evaluated at $x=\pi / 3$, for different values of $\rho_{i}$ (f). Contour plot of the magnetic island separatrix at the corresponding time have been superimposed (white line). The plots refer to the normalized time $\gamma_{L} t=5.5$

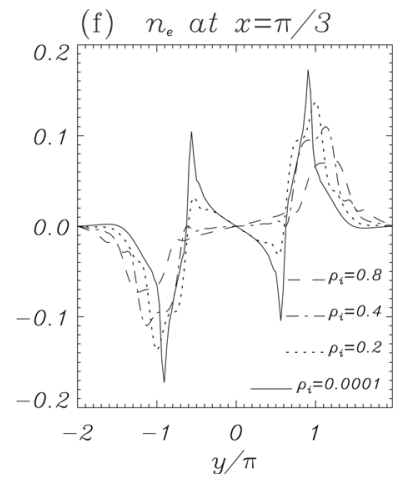

This equation still retains the form of an advection equation for an infinity of Lagrangian invariants $f+\left(1 / d_{i}\right)\left(v_{\|} / v_{t i}^{2}\right) F_{0}\langle A\rangle$ labelled by the value of the parallel velocity and of the magnetic moment. Remark that the advecting stream functions correspond namely to $\Phi_{ \pm}$, when $v_{\|}= \pm v_{t i}$ and $\langle\phi\rangle$ and $\langle A\rangle$ are replaced by $\Phi$ and $\mathcal{A}$, respectively. Therefore, the gyrokinetic origin of $\Phi_{ \pm}$can be traced back to the stream function $\langle\phi\rangle-v_{\|}\langle A\rangle$, which reflects the motion of gyrocenters due to the gyroaveraged $\mathbf{E} \times \mathbf{B}$ flow, as well as their free streaming along the gyroaveraged magnetic field. The reason why the values $v_{\|}= \pm v_{t i}$ are those selected in the four-field gyrofluid model must be a consequence of the isothermal closure. Indeed, Eqs. (2)-(3) are obtained from the first two moments of Eq. (35) imposing $p_{\|}=\tau n_{i}$, where $p_{\|}$is the parallel pressure. This condition translates into

$$
\int d v_{\|} d \mu v_{\|}^{2} f=\int d v_{\|} d \mu v_{t i}^{2} f
$$

Such closure can be formally obtained by a distribution function of the form

$$
\begin{aligned}
f\left(x, y, v_{\|}, t ; \mu\right)= & f_{+}(x, y, t ; \mu) \delta\left(v_{\|}-v_{t i}\right) \\
& -f_{-}(x, y, t ; \mu) \delta\left(v_{\|}+v_{t i}\right) .
\end{aligned}
$$

From Eq. (37), it is clear how the values $v_{\|}= \pm v_{t i}$ are selected in the isothermal closure.

\section{B. Role of the topological invariants of the system}

Equations (29) and (30) tell us that the topology of the fields $I_{ \pm}$and $G_{ \pm}$is preserved during the evolution of the system. These conservation laws set a substantial constraint on the dynamics of the collisionless magnetic reconnection, and 
they provide a framework for interpreting the spatial structures of the original fields of the model.

Considering Eqs. (29), we note that since the stream functions $\Phi_{ \pm}$correspond to velocity fields $\mathbf{v}_{ \pm}$that rotate in opposite directions, the fields $I_{ \pm}$, advected by such velocity fields, get then stretched accordingly. Indeed, from Fig. 7, we see that the initial topology of the contour lines of $I_{+}$is preserved (the same happens for $I_{-}$, not shown here), while they are stretched, similarly to what was first observed for the invariants $G_{ \pm}$(Refs. 25, 26, and 33).

From the relations

$$
\mathcal{A}+d_{i}^{2} u_{i}=\frac{I_{+}+I_{-}}{2}, \quad d_{i} \rho_{i} n_{i}=\frac{I_{+}-I_{-}}{2},
$$

we can deduce that the stretching process reflects also on the fields $n_{i}$ and $D=\mathcal{A}+d_{i}^{2} u_{i}$, which tend to exhibit structures that become thinner with time. Because $\mathcal{A}=\nabla^{-2} \Gamma_{0}^{1 / 2} u_{e}$ $-\nabla^{-2} \Gamma_{0} u_{i}$, we also infer that $\mathcal{A}$ will have in general "smoother" gradients, compared to $u_{i}$.

For $\rho_{i} \rightarrow 0$, we obtain $I_{ \pm} \rightarrow D$ and $\Phi_{ \pm} \rightarrow \phi$, and thus, the stretching in opposite directions of $I_{+}$and $I_{-}$does not occur because the magnetic contribution $\rho_{i} \mathcal{A} / d_{i}$ to the velocity fields $\mathbf{v}_{ \pm}$gets suppressed. This can be seen from Fig. 8 when comparing the contour plots of $I_{+}$for different values of $\rho_{i}$.

In the cold ion limit, because of the Lagrangian conservation of $D$, the ion guiding center velocity that develop near the $\mathrm{X}$-point is advected by $\phi$ along the neutral line, leading to the formation of bar-shaped layers, as shown in Fig. 3(a). Furthermore, from Fig. 8, we note the broadening of the contour lines of $I_{ \pm}$near the X-point for large $\rho_{i}$, which reflects on the scaling relation for the width $\delta_{u_{i}}$.

The evolution of the invariants $G_{ \pm}$and their dependence on $\rho_{i}$ do not differ much from those of the three-field gyromodel, ${ }^{15}$ and they typically undergo phase mixing. This reflects on the structures of $n_{e}$ and $F=A-d_{e}^{2} u_{e}$ by virtue of the following relations:

$$
A-d_{e}^{2} u_{e}=\frac{G_{+}+G_{-}}{2}, \quad d_{e} \rho_{s} n_{e}=\frac{G_{+}-G_{-}}{2} .
$$

The phase-mixing picture is valid for both cold and hot ion regimes. However, as already observed in the three-field model of Ref. 15, increasing ion temperature results in smoother gradients of $G_{ \pm}$inside the island (Fig. 9), leading
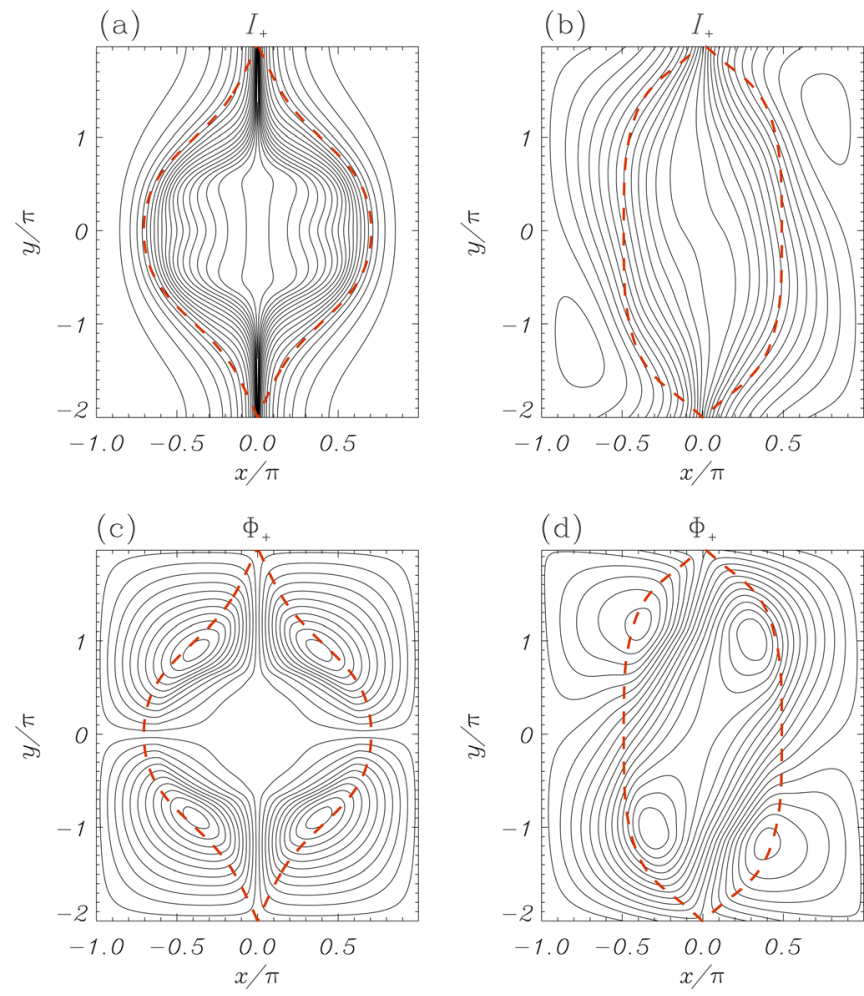

FIG. 8. Contour plots of $I_{+}$for $\rho_{i}=10^{-4}$ (a) and $\rho_{i}=0.8$ (b). Contour plots of $\Phi_{+}$for $\rho_{i}=10^{-4}$ (c) and $\rho_{i}=0.8$ (d). The magnetic island separatrix at the corresponding time have been superimposed (red dashed line). The plots refer to the normalized time $\gamma_{L} t=5.5$.

to a more uniform electron density in the island core, as shown in Figs. 6(d)-6(f).

We note that, since the rotation of $\overline{\mathbf{v}}_{ \pm}$and $\mathbf{v}_{ \pm}$depends on the electric charge of ions and electrons, then the fields $I_{ \pm}$ rotate in opposite directions with respect to $G_{ \pm}$, as we may notice by comparing Figs. 8 and 9 .

For $\rho_{i} \simeq \rho_{s}$, due to the small electron/ion mass ratio, the magnetic component present in the stream functions $\Phi_{ \pm}$, which is inversely proportional to $d_{i}$, gives a smaller contribution with respect to the magnetic component in $\phi_{ \pm}$, which is inversely proportional to $d_{e}$. Consequently, in general, the structures of $\Phi_{ \pm}$remain closer to those of $\Phi$, whereas the structures of $\phi_{ \pm}$are greatly influenced by the magnetic island shape, coming from the contribution due to $A$. This reflects, of course, on the different ways in which $I_{ \pm}$and $G_{ \pm}$get
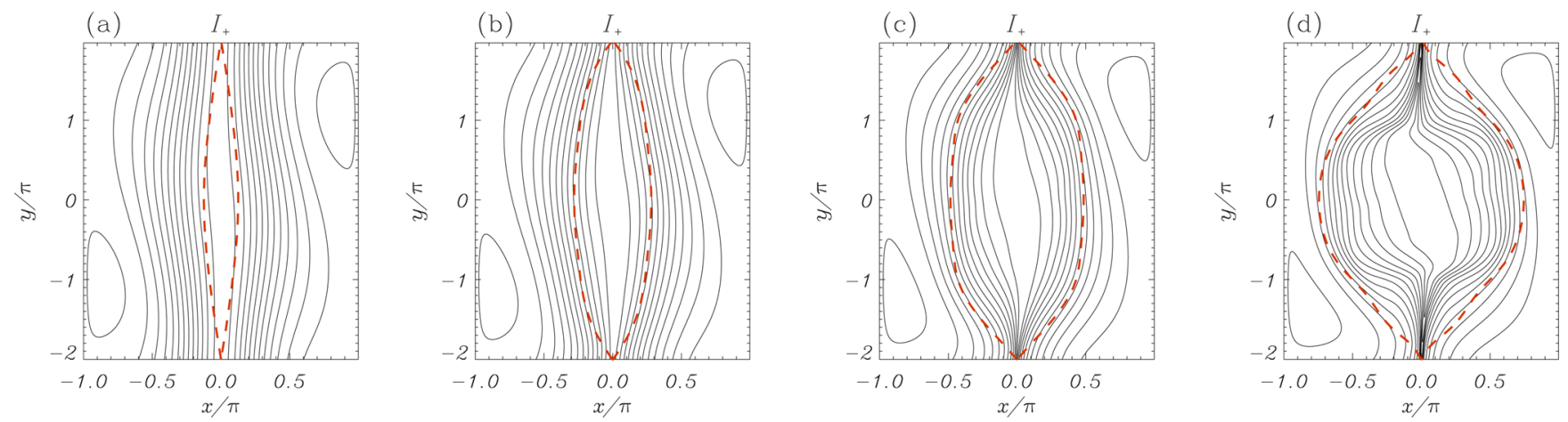

FIG. 7. Contour plots of $I_{+}$for $\rho_{i}=0.4$ at $\gamma_{L} t=2(\mathrm{a}), \gamma_{L} t=3.5$ (b), $\gamma_{L} t=5$ (c), $\gamma_{L} t=6.5$ (d). The magnetic island separatrix at the corresponding time have been superimposed (red dashed line). 

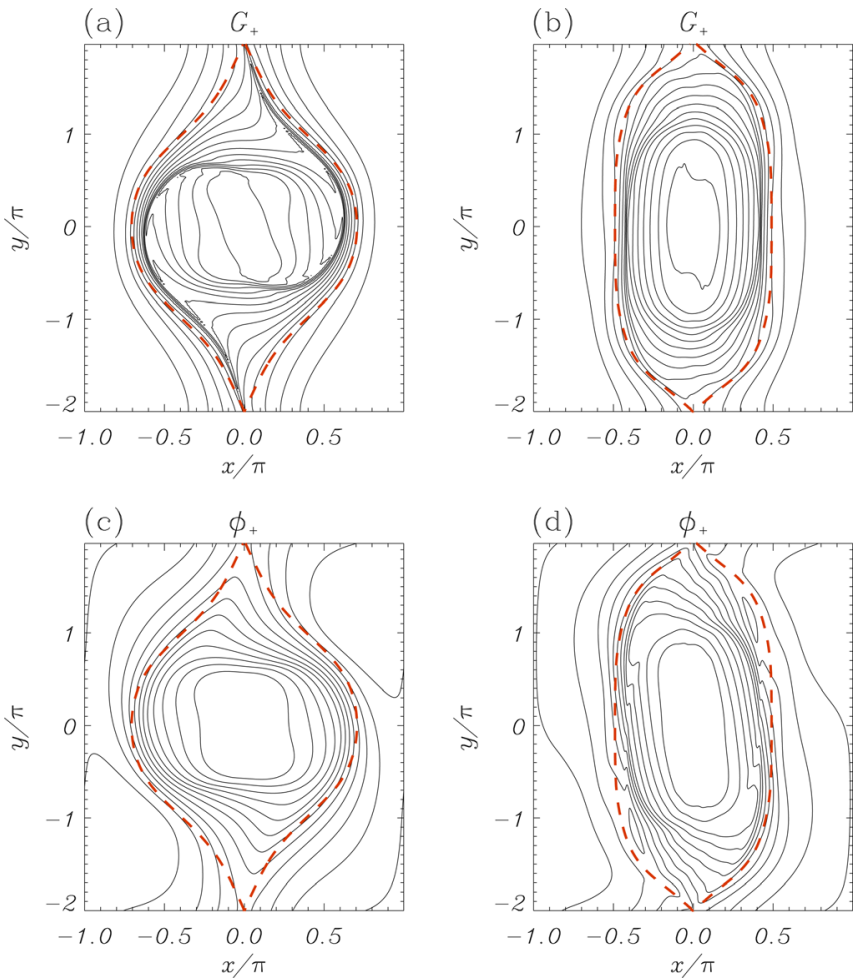

FIG. 9. Contour plots of $G_{+}$for $\rho_{i}=10^{-4}$ (a) and $\rho_{i}=0.8$ (b). Contour plots of $\phi_{+}$for $\rho_{i}=10^{-4}$ (c) and $\rho_{i}=0.8$ (d). The magnetic island separatrix at the corresponding time have been superimposed (red dashed line). The plots refer to the normalized time $\gamma_{L} t=5.5$.

advected by the corresponding stream functions. We remark that, in the parent gyro (drift)-kinetic models, a similar feature concerns the terms quadratic in $\mathcal{A}(A)$ in the stream functions that advect the gyro (drift)-kinetic distribution functions at fixed canonical momentum (consider, for instance, the expression for $\bar{\varphi}$ in Eq. (33) for the gyrokinetic case).

\section{ANALYSIS OF THE ENERGY EVOLUTION}

One of the fundamental characteristics of magnetic reconnection is the rapid conversion of magnetic energy into thermal, kinetic, and fast particle energy. Hence, it is important to examine how the initial free energy is released into the different channels. Here, we exclude considerations on the particle acceleration problem, since we are dealing with a purely fluid description of the plasma.

The total energy of the four-field gyrofluid model corresponds to the following Hamiltonian functional:

$H=\frac{1}{2} \int d^{2} x\left(\tau \rho_{s}^{2} n_{i}^{2}+\rho_{s}^{2} n_{e}^{2}+d_{i}^{2} u_{i}^{2}+d_{e}^{2} u_{e}^{2}+|\nabla A|^{2}+\Phi n_{i}-\phi n_{e}\right)$.

The terms in Eq. (40) represent, respectively, the ion and electron thermal energies $\left(E_{t h_{i}}\right.$ and $\left.E_{t h_{e}}\right)$, the parallel ion and electron kinetic energies $\left(E_{k_{i}}\right.$ and $\left.E_{k_{e}}\right)$, the magnetic energy $\left(E_{B}\right)$ and the electrostatic energy of the ions $\left(E_{e l_{i}}\right)$ and electrons $\left(E_{e l_{e}}\right)$.

From Fig. 10, we can see that only a small amount of the total energy (less than $1.5 \%$ ) is dissipated numerically at the end of the simulations. In particular, the decrease rate of the total energy is much less than that of the magnetic energy.
This indicates that the magnetic energy decline is due to a transformation (in principle reversible) into other forms of energy and not to numerical dissipation of the total energy.

We first observe that the amount of $E_{B}$ converted into other forms of energy during the reconnection process is about the same for $\rho_{i}=10^{-4}$ and $\rho_{i}=0.8$. However, the way in which this energy is transformed is quite different in the two cases. In the cold ion regime, the reconnection process converts $E_{B}$ initially mainly into $E_{t h_{e}}$. For $\gamma_{L} t>4$, the increase in $E_{e_{e}}$ prevails and it eventually remains comparable to that of $E_{t h_{e}}$. On the other hand, in the hot ion regime, $E_{B}$ is transformed mainly into $E_{e l_{e}}$. Since we have seen from Fig. 6 that, in the hot ion regime, the fluctuations of $n_{e}$ have lower amplitude, it is expected that $\delta E_{t h_{e}, \rho_{i}=0.8}$

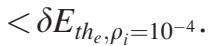

Then, we compare the following approximate expressions obtained for $E_{e l_{e}}$ using the quasi-neutrality condition (13) in the cold ion limit:

$$
\rho_{i} \rightarrow 0 \Rightarrow E_{e l_{e}} \approx-\frac{1}{2} \int d^{2} x n_{e} \nabla^{-2} n_{e} .
$$
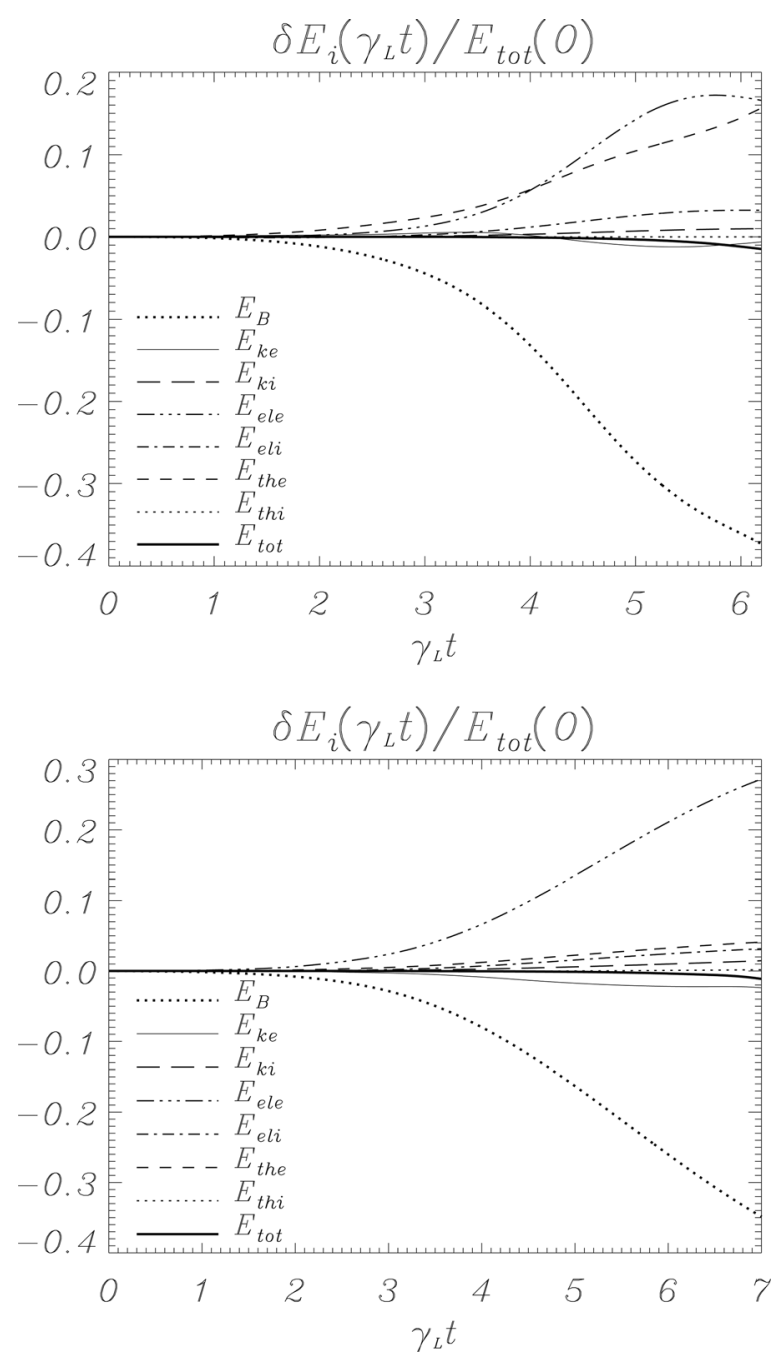

FIG. 10. Time variations of the energy components with respect to their initial value, expressed as $\delta E_{i}\left(\gamma_{L} t\right) / E_{t o t}(0)$, for $\rho_{i}=10^{-4}$ (top) and $\rho_{i}=0.8$ (bottom). 
The contribution of $n_{i}$ is neglected since we observed that $n_{e} \gg n_{i}$. In the opposite limit $\rho_{i} \rightarrow \infty$, the relation (20) approximately holds (for our simulations even the largest value of $\rho_{i}$ is smaller than 1 , but because $\rho_{i}$ is always multiplied times differential operators, this behaviour is observed when the small scales of the strong nonlinear phase are formed). Therefore, we have

$$
\rho_{i} \rightarrow \infty \quad \Rightarrow \quad E_{e l_{e}} \approx \frac{1}{2} \int d^{2} x \rho_{i}^{2} n_{e}^{2}
$$

where, also in this case, we assumed $n_{i} \ll n_{e}$. The smaller scale structures of the electron density in the cold ion limit reflect on the spectral distribution, which is concentrated in higher wave vectors than in the hot ion regime. Due to the presence of the operator $\nabla^{-2}$ in (41), this leads to $\delta E_{e l_{e}, \rho_{i}=0.8}>\delta E_{e l_{e}, \rho_{i}=10^{-4}}$, in spite of the larger amplitude of $n_{e}$ for cold ions.

The relatively small fraction of energy going into $E_{k_{i}}$ in both cases reflects the relatively small amplitude of the fluctuations of $u_{i}$. Analogously, $\delta E_{t h_{i}}$ grows little even for large $\rho_{i}$, due to the modest amplitude of the fluctuations in $n_{i}$.

\section{CONCLUSIONS}

We investigated plasma and magnetic dynamics by means of a gyrofluid model which allows for magnetic reconnection mediated by electron inertia. Unlike its closest predecessor, the three-field gyrofluid model of Refs. 14 and 15 , the system studied here, accounts also for parallel ion guiding center flow. The presence of such flow, in turn, drives the evolution of the ion guiding center density, which was purely advected in the three-field model. Our analysis reveals that, in the cold ion limit, $n_{i}$ and $u_{i}$ develop thin layers, with opposite parities with respect to the $x=0$ axis. An analogous feature was observed in the parallel flow and generalized vorticity, respectively, of the Fitzpatrick-Porcelli model, to which the present gyrofluid model can be mapped in the limit $\beta_{e} \ll 1, \rho_{i} \rightarrow 0, d_{e} / d_{i} \rightarrow 0$. Adding ion temperature effects results in a broadening of these layers.

Due to the low- $\beta$ limitation of the gyrofluid model, however, we observed that the amplitudes of the fluctuations in $u_{i}$ and $n_{i}$ remain small, when compared to those of $n_{e}$ and $u_{e}$. The dynamics of the latter quantities, indeed, does not differ much from that observed in the three-field model.

The cusp-like profiles of the parallel current density at the X-point, a well known characteristic of collisionless reconnection, have been observed to decrease their amplitude when $\rho_{i}$ increases.

Concerning the magnetic dynamics, we observed that ion temperature modifies the island shape by increasing the angle between the separatrix and the $y$ axis and yielding more elongated islands. The spectrum of the magnetic flux function suggests that this could be due to higher- $m$ modes becoming relevant in the hot ion regime.

The structures in the density and velocity fields have been interpreted in terms of the invariants $I_{ \pm}$and $G_{ \pm}$. Here, the main novelty resides in the difference between the behavior of $I_{ \pm}$and $G_{ \pm}$, due to the small mass ratio and the opposite charge of electrons and ions. Indeed, the fields $\overline{\mathbf{v}}_{ \pm}$are much less affected by the contribution of the magnetic potential and consequently advect $I_{ \pm}$differently than the way $\mathbf{v}_{ \pm}$advect $G_{ \pm}$. This explains the qualitatively different structures forming in electron and ion-related fields.

Finally, similarly to $G_{ \pm}$and $\mathbf{v}_{ \pm}$, also $I_{ \pm}$and $\overline{\mathbf{v}}_{ \pm}$have been shown to possess counterparts at the gyrokinetic level.

\section{ACKNOWLEDGMENTS}

The authors gratefully acknowledge valuable discussions with Dario Borgogno. This work was supported by the European Community under the contracts of Association between EURATOM and ENEA and between EURATOM, CEA, and the French Research Federation for fusion studies. F.L.W. was supported by the U.S. Department of Energy under Contract No. DE-FG02-04ER-54742. The views and opinions expressed herein do not necessarily reflect those of the European Commission. Financial support was also received from the Agence Nationale de la Recherche (ANR GYPSI n. 2010 BLAN 941 03).

${ }^{1}$ E. R. Priest and T. G. Forbes, Magnetic Reconnection: MHD Theory and Applications (Cambridge University Press, 2000).

${ }^{2}$ D. Biskamp, Magnetic Reconnection in Plasmas (Cambridge University Press, 2000).

${ }^{3}$ D. Del Sarto, F. Califano, and F. Pegoraro, Mod. Phys. Lett. B 20, 931 (2006).

${ }^{4}$ E. Tassi, P. J. Morrison, D. Grasso, and F. Pegoraro, Nucl. Fusion 50, 034007 (2010).

${ }^{5}$ A. J. Brizard, Phys. Fluids B 4, 1213 (1992).

${ }^{6}$ W. Dorland and G. W. Hammett, Phys. Fluids B 5, 812 (1993).

${ }^{7}$ M. A. Beer and G. W. Hammett, Phys. Plasmas 3, 4046 (1996).

${ }^{8}$ B. Scott, Phys. Plasmas 7, 1845 (2000).

${ }^{9}$ P. B. Snyder and G. W. Hammett, Phys. Plasmas 8, 3199 (2001).

${ }^{10}$ D. Strintzi, B. D. Scott, and A. J. Brizard, Phys. Plasmas 12, 052517 (2005).

${ }^{11}$ B. Scott, Phys. Plasmas 17, 102306 (2010).

${ }^{12}$ D. Grasso, F. Califano, F. Pegoraro, and F. Porcelli, Plasma Phys. Rep. 26, 512 (2000)

${ }^{13}$ D. Del Sarto, C. Marchetto, F. Pegoraro, and F. Califano, Plasma Phys. Controlled Fusion 53, 035008 (2011).

${ }^{14}$ F. L. Waelbroeck, R. D. Hazeltine, and P. J. Morrison, Phys. Plasmas 16, 032109 (2009)

${ }^{15}$ D. Grasso, E. Tassi, and F. L. Waelbroeck, Phys. Plasmas 17, 082312 (2010).

${ }^{16}$ E. Tassi, F. L. Waelbroeck, and D. Grasso, J. Phys.: Conf. Ser. 260, 012020 (2010).

${ }^{17}$ F. L. Waelbroeck and E. Tassi, Commun. Nonlinear Sci. Numer. Simul. 17, 2171 (2012).

${ }^{18}$ A. Biancalani and B. D. Scott, Europhys. Lett. 97, 15005 (2012).

${ }^{19}$ S. Vincena, W. Gekelman, and J. Maggs, Phys. Rev. Lett. 93, 105003 (2004).

${ }^{20}$ X. yu Wang, X. yi Wang, Z. xing Liu, and Z. yuan Li, Phys. Plasmas 5, 4395 (1998).

${ }^{21}$ B. N. Rogers, R. E. Denton, J. F. Drake, and M. A. Shay, Phys. Rev. Lett. 87, 195004 (2001).

${ }^{22}$ R. Fitzpatrick and F. Porcelli, Phys. Plasmas 11, 4713 (2004); R. Fitzpatrick and F. Porcelli, Phys. Plasmas 14, 049902 (2007).

${ }^{23}$ H. P. Furth, J. Killeen, and M. N. Rosenbluth, Phys. Fluids 6, 459 (1963).

${ }^{24}$ F. Porcelli, Phys. Rev. Lett. 66, 425 (1991).

${ }^{25}$ E. Cafaro, D. Grasso, F. Pegoraro, F. Porcelli, and A. Saluzzi, Phys. Rev. Lett. 80, 4430 (1998).

${ }^{26}$ D. Grasso, F. Califano, F. Pegoraro, and F. Porcelli, Phys. Rev. Lett. 86, 5051 (2001).

${ }^{27}$ A. Y. Aydemir, Phys. Fluids B 4, 3469 (1992).

${ }^{28}$ X. Wang and A. Bhattacharjee, Phys. Rev. Lett. 70, 1627 (1993).

${ }^{29}$ M. Ottaviani and F. Porcelli, Phys. Rev. Lett. 71, 3802 (1993).

${ }^{30} \mathrm{X}$. Wang and A. Bhattacharjee, Phys. Plasmas 2, 171 (1995).

${ }^{31}$ M. Ottaviani and F. Porcelli, Phys. Plasmas 2, 4104 (1995).

${ }^{32}$ B. Rogers and L. Zakharov, Phys. Plasmas 3, 2411 (1996). 
${ }^{33}$ D. Grasso, F. Pegoraro, F. Porcelli, and F. Califano, Plasma Phys. Controlled Fusion 41, 5051 (1999).

${ }^{34}$ A. Bhattacharjee, K. Germaschewski, and C. S. Ng, Phys. Plasmas 12, 042305 (2005).

${ }^{35}$ F. L. Waelbroeck, Phys. Rev. Lett. 70, 3259 (1993).

${ }^{36}$ J. F. Drake and R. G. Kleva, Phys. Rev. Lett. 66, 1458 (1991).

${ }^{37}$ J. F. Drake, R. G. Kleva, and M. E. Mandt, Phys. Rev. Lett. 73, 1251 (1994).

${ }^{38}$ D. Biskamp, E. Schwarz, and J. F. Drake, Phys. Rev. Lett. 75, 3850 (1995).

${ }^{39}$ D. Biskamp, E. Schwarz, and J. F. Drake, Phys. Plasmas 4, 1002 (1997).

${ }^{40}$ B. Scott and F. Porcelli, Phys. Plasmas 11, 5468 (2004).

${ }^{41}$ D. Grasso, D. Borgogno, F. Pegoraro, and E. Tassi, Nonlinear Processes Geophys. 16, 241 (2009).

${ }^{42}$ E. Tassi, D. Grasso, and F. Pegoraro, Commun. Nonlinear Sci. Numer. Simul. 15, 2 (2010).
${ }^{43}$ D. Del Sarto, F. Califano, and F. Pegoraro, Phys. Rev. Lett. 91, 235001 (2003).

${ }^{44}$ D. Del Sarto, F. Califano, and F. Pegoraro, Phys. Plasmas 12, 012317 (2005).

${ }^{45}$ H. J. de Blank, Phys. Plasmas 8, 3927 (2001).

${ }^{46}$ T. J. Schep, F. Pegoraro, and B. N. Kuvshinov, Phys. Plasmas 1, 2843 (1994).

${ }^{47}$ T. V. Liseikina, F. Pegoraro, and E. Yu. Echkina, Phys. Plasmas 11, 3535 (2004).

${ }^{48}$ F. Pegoraro, T. Liseikina, and E. Yu. Echkina, Transp. Theory Stat. Phys. 34, 243 (2005).

${ }^{49}$ F. Pegoraro, T. Liseikina, and E. Yu. Echkina, Phys. Scr. T116, 88 (2005).

${ }^{50}$ A. Zocco and A. A. Schekochihin, Phys. Plasmas 18, 102309 (2011). 